\title{
Uso de las TIC en
} estudiantes de quinto y sexto grado de educación primaria

Marisol Villegas Pérez*

Sonia Verónica Mortis Lozoya**

Ramona Imelda García López***

Elizabeth del Hierro Parra****

\section{RESUMEN}

El propósito de este estudio es identificar la percepción de los alumnos de quinto y sexto de educación primaria, en escuelas públicas, sobre sus competencias en el uso de las tecnologías de la información y la comunicación (TIC) e indagar si existen diferencias en cuanto al sexo y a tener computadora y acceso a internet en casa. La metodología fue cuantitativa no experimental transeccional y la muestra se conformó de 201 estudiantes de escuelas primarias públicas: $51.6 \%$ corresponde a alumnos de quinto y 48.4, de sexto; de estos, 52.6 eran niños y 47.4, niñas. Los instrumentos empleados fueron dos encuestas con escala tipo Likert con cinco opciones de respuesta. La primera encuesta midió el uso de las TIC en la vida diaria y la segunda, el uso de las TIC en la escuela. Los resultados muestran que los estudiantes hacen uso moderado de las TIC y no hay diferencias significativas en cuanto al sexo y a tener computadora en casa, o no, pero sí en el acceso a internet. El estudio concluye que los alumnos utilizan las TIC más para actividades de esparcimiento que para labores escolares y que sus competencias al respecto son limitadas.

Palabras clave:

Estudiantes de primaria, uso de las TIC, tecnologías en la escuela.

* Maestra en Investigación Educativa. Profesora auxiliar de licenciatura y adjunta de maestría en el Departamento de Educación del Instituto Tecnológico de Sonora.

** Doctora en Educación. Profesora-investigadora del Departamento de Educación del Instituto Tecnológico de Sonora.

*** Doctora en Educación. Profesora-investigadora del Departamento de Educación del Instituto Tecnológico de Sonora.

**** Doctora en Educación. Profesora-investigadora del Departamento de Educación del Instituto Tecnológico de Sonora. 


\section{Use of ICT in 5th and 6th grade students of elementary school}

\section{Abstract}

The purpose of this study is to identify the perception of students in fifth and sixth grade public elementary schools on their competencies in the use of Information and Communication Technology (ICT) and investigate whether there are differences based on gender, have a computer and Internet access at home. A non-experimental quantitative methodology transactional was used and the sample was composed of $201 \mathrm{stu}-$ dents from public elementary schools where $51.6 \%$ are fifth- and sixth $48.4 \%$ of which $52.6 \%$ were boys and $47.4 \%$ girls. The instruments used were two surveys Likert scale with five response options. The first survey measures the use of ICT in daily life and the second survey measures the use of ICT in school. The results show that students make moderate use of ICT and that there are no significant differences based on gender and have or not have a computer but do exist in access to the Internet. It is concluded that the students use ICT more for recreation than for school activities and their competencies in the matter are limited.

Keywords:

Elementary students, use of ICT, technologies in the school.

\section{INTRODUCCIÓN}

$\mathrm{E}^{\mathrm{n}}$ la actualidad se ha debatido bastante sobre la importancia de incorporar las tecnologías de la información y la comunicación (TIC) a la educación, ya que a través de ellas se promueve el aprendizaje, porque propician la construcción activa y participativa del conocimiento de los estudiantes; tienen la función de ser un medio de comunicación e intercambio de conocimiento y experiencias, además de ser un instrumento para procesar información, fuente de recursos, espacios educativos y desarrollo cognitivo. Esto se debe a que el uso de las TIC supone el considerar las posibilidades didácticas que ofrecen para facilitar el proceso de enseñanza-aprendizaje en función del contexto 
... la incorporación de las TIC en la educación contribuye a que los estudiantes desarrollen la competencia digital que implica ser una persona autónoma, eficaz, responsable, crítica y reflexiva al seleccionar, tratar y utilizar la información y sus fuentes, además de sus herramientas tecnológicas

(Area, 2009).

en el aula, las características del alumno, los propósitos y los contenidos educativos; asimismo, posibilitan que el profesor se convierta en un guía que orienta al alumno respecto a su aprendizaje al permitirle ser el protagonista de la clase y trabajar de manera autónoma y en colaboración con sus compañeros (Gómez y Macedo, 2010; Castañeda, Carrillo y Quintero, 2013).

De igual manera, la incorporación de las TIC en la educación contribuye a que los estudiantes desarrollen la competencia digital que implica ser una persona autónoma, eficaz, responsable, crítica y reflexiva al seleccionar, tratar y utilizar la información y sus fuentes, además de sus herramientas tecnológicas (Area, 2009). Revuelta (2011) explica que “... la competencia digital se sustenta en las competencias básicas en materia de TIC: el uso de ordenadores para obtener, evaluar, almacenar, producir, presentar e intercambiar información y comunicarse $\mathrm{y}$ participar en redes de colaboración a través de internet" (p. 3). La competencia digital entraña el uso seguro y crítico de las tecnologías de la sociedad de información para el trabajo, el ocio y la comunicación. Se sustenta en las competencias básicas en TIC: el uso de ordenadores para obtener, evaluar, almacenar, producir, presentar e intercambiar información, y comunicarse y participar en redes de colaboración a través de internet (European Commission, 2007).

Debido a la importancia de desarrollar la competencia digital en estudiantes y de lo que deberían saber y ser capaces de hacer para aprender efectivamente y vivir en un mundo cada vez más digital, diferentes organismos expertos en el área han establecido modelos y estándares TIC clasificados en distintas categorías para facilitar su uso. Algunos de estos estándares han sido propuestos por la Sociedad Internacional para la Tecnología en la Educación de Canadá (ISTE, por sus siglas en inglés) y el proyecto Estándares Nacionales de Tecnologías Educativa para Estudiantes de los Estados Unidos (NETS-S), los cuales fueron retomados en el Programa de Habilidades Digitales para Todos de la Secretaría de Educación Pública (SEP) en México: creatividad e innovación; comunicación y colaboración; investigación y manejo de información; pensamiento crítico; ciudadanía digital; y funcionamiento y conceptos de las TIC (NETS-S, 2007; ISTE, 2007; SEP, 2011).

Dichos estándares son, según ISTE (2007):

- Creatividad e innovación, que implica demostrar el pensamiento creativo, el desarrollo de productos y los procesos innovadores utilizando las TIC y la construcción de conocimiento. 
- Comunicación y colaboración, en las que se requiere la utilización de medios y entornos digitales que les permitan comunicar ideas e información a una audiencia múltiple, interactuar con otros y trabajar de forma colaborativa.

- Investigación y manejo de información, que implica la aplicación de herramientas digitales que permitan a los alumnos recabar, seleccionar, analizar, evaluar y utilizar información, procesar datos y comunicar resultados.

- Pensamiento crítico, que requiere el desarrollo de habilidades de pensamiento crítico para planear, organizar y llevar a cabo investigaciones, administrar proyecto, resolver problemas y tomar decisiones sustentadas en información, utilizando herramientas digitales.

- Ciudadanía digital, que precisa la comprensión de asuntos humanos, culturales y sociales relacionados con el uso de las TIC y la aplicación de conductas éticas, legales, seguras y responsables en su uso.

- Funcionamiento y conceptos de las TIC, que implica la comprensión de conceptos, sistemas y funcionamiento de estas para seleccionarlas y utilizarlas de manera productiva y transferir el conocimiento existente al aprendizaje de nuevas TIC.

Por la relevancia del uso de las TIC y el desarrollo de las competencias digitales que deben poseer todos los actores participantes en el proceso de enseñanzaaprendizaje, principalmente en educación básica, diferentes autores han realizado estudios para analizar y medir su efecto. Quintana, Cortada y Riera (2012) identificaron que los estudiantes de primaria y secundaria, que tienen un mayor contacto con las TIC, saben cómo usar la tecnología, pero no presentaron diferencias para la búsqueda de información en internet. Además, todos estos estudiantes muestran un mejor conocimiento y control de la computadora, así como mejores habilidades de alfabetización web.

Por su parte, Ortiz, Peñaherrera y Ortega (2012) encuestaron a estudiantes de quinto y sexto de primaria con la finalidad de conocer en profundidad cómo se da la incorporación de las TIC en la escuela. Algunos de los resultados señalan que el alumno hace uso de la computadora sobre todo en función del juego, después para trabajos en clase y, por último, para navegar en internet; además, todos los alumnos utilizan la computadora en la escuela y con mayor frecuencia en las asignaturas de lengua extranjera y matemáticas.

Aesaert y Van Braak (2015) investigaron la relación entre el sexo de los estudiantes y sus competencias TIC y demuestran que las niñas tienen mayor dominio que los niños. Por otra parte, el estudio de McKenney y Voogt (2010), que examina el acceso, percepciones y uso de la tecnología dentro y fuera del entorno escolar de los niños pequeños de los Países Bajos, no encontró diferencia en el sexo en cuanto al uso de la computadora ni en el nivel académico, a pesar de que los niños mostraron actitudes más positivas que las niñas.

\section{... resultados señalan que \\ el alumno hace uso de la} computadora sobre todo en función del juego, después para trabajos en clase y, por último, para navegar en internet... 
Asimismo, en los estudios efectuados por Volman, Van Eck, Heemskerk y Kuiper (2005), con alumnos de familias con antecedentes de minoría étnica y de la población mayoritaria en los Países Bajos, descubrieron que en la educación primaria las diferencias entre los niños y las niñas parecían ser pequeñas; en la educación secundaria, la actitud de las niñas hacia las computadoras parece ser menos positiva que la de los niños. Hatlevik (2012) examinó los factores que afectan el uso productivo de las computadoras en la escuela en los estudiantes de noveno grado; sus resultados demuestran que la educación de los padres influye en el rendimiento de los alumnos con y sin equipos; en general, las niñas reportan mayores niveles de uso productivo de las computadoras en comparación con los niños, lo que indica que las niñas tienen éxito en la escuela mediante el uso de las computadoras en sus clases.

En el estudio realizado por Chávez, Cantú y Rodríguez (2016), para determinar el desempeño obtenido por los estudiantes de cuarto, quinto y sexto grado, al introducir las TIC para el desarrollo de las competencias digitales, tratamiento de la información y trabajo en equipo, se identificó que los estudiantes se ubicaron en el nivel medio alto en las competencias digitales y en el manejo o tratamiento de la información y alto en la competencia de trabajo en equipo. Por otra parte, Fajardo, Villalta y Salmerón (2016) efectuaron una investigación con estudiantes de quinto de primaria y tercero de secundaria. Los resultados muestran que los alumnos de secundaria obtuvieron mayores puntuaciones que los de primaria en cuanto a las habilidades digitales, comprensión lectora y lectura digital; también, que entre más practican tareas de lectura digital, los estudiantes incrementan sus habilidades digitales y su comprensión lectora en papel.

Por otra parte, en México se llevó a cabo una investigación cuya intención fue medir las competencias tecnológicas de estudiantes de secundaria del municipio de Cajeme; se encontró que los 
alumnos inscritos en escuelas privadas se perciben más competentes que los de las públicas. Los estudiantes que cuentan con computadora e internet en sus hogares dominan tres de las seis competencias propuestas por los estándares ISTE: comunicación y colaboración, investigación y manejo de la información, y funcionamiento y conceptos de TIC (Mortis, Cuevas, García y Cabero, 2014).

\section{PLANTEAMIENTO DEL PROBLEMA}

En México, la SEP ha hecho varios esfuerzos por la incorporación de las TIC en educación básica mediante la implementación de distintos programas:

- El programa Computación Electrónica en Educación Básica apoyó con talleres y laboratorios de computación e informática (1985).

- En 1996 se implementó la propuesta tecnológica Red Escolar, que promovía el trabajo colaborativo, así como la investigación y el intercambio de ideas.

- En el programa de Enciclomedia (2004) los grupos de quinto y sexto grado fueron equipados de herramientas digitales.

- Habilidades Digitales para Todos promovió el uso eficiente de la tecnología en educación básica (2009), que se basa en los estándares propuestos por la ISTE y los NETS-S.

- En 2013, el programa Mi Compu.Mx entregó una computadora a cada alumno de quinto y sexto grado de primaria en escuelas públicas. En 2014, cambió de nombre a Programa de Inclusión y Alfabetización Digital, el cual asigna una tableta electrónica a los estudiantes de dichos grados (SEP, 2013).

Cabe destacar que estos programas fueron propuestos sin fines de lucro, con la intención de reducir la brecha digital y promover una cultura de ciudadanía digital; sin embargo, su principal actividad ha consistido en equipar las instituciones y la dotación de recursos tecnológicos para los estu- diantes de educación primaria sin un diagnóstico previo sobre la aceptación, el conocimiento y uso de las TIC. A partir de 2014, un grupo de investigadores de los estados participantes en el programa Mi Compu.Mx: Sonora, Colima, Tabasco y Nuevo León, han analizado el impacto de la entrega gratuita de equipos y han hecho hincapié en las experiencias de los actores que participan en el uso de los recursos del Programa de Inclusión y Alfabetización Digital. Entre los principales hallazgos destaca la falta de formación para docentes y las pocas habilidades digitales de los estudiantes de educación primaria (Díaz, Rodríguez, Sánchez, Rivera y Ramírez, 2015; García, Angulo y Cuevas, 2015; Rocha y Ramírez, 2015).

En ese sentido, es importante conocer el desarrollo de la competencia en el uso de las TIC de los estudiantes de quinto y sexto grado mediante el empleo y aprovechamiento de la computadora o tableta electrónica, ya que la competencia digital está relacionada con el uso habitual de los medios y recursos didácticos digitales, así como de las TIC en forma segura y crítica; a través de internet y las computadoras pueden obtener, evaluar, almacenar, producir, presentar e intercambiar información (Trigueros, Sánchez y Vera, 2012; Parlamento Europeo, 2009).

Por lo anterior, se pretende conocer la percepción de los estudiantes de quinto y sexto de primaria respecto a sus competencias en el uso de las TIC, así como las diferencias en la percepción del desarrollo de dichas competencias entre niñas y niños, y disponer de computadora, o no, y acceso a internet en casa; esto tiene el propósito de recabar información que brinde un panorama general sobre los requerimientos de los estudiantes que le permitan aprovechar al máximo los recursos propuestos por el PIAD y, por ende, fortalecer sus competencias en el uso de tecnología.

\section{Objetivos}

Identificar la percepción de los alumnos de quinto y sexto de primaria sobre sus competencias en el 
uso de las TIC a fin de proponer estrategias que le ayuden a fortalecer o desarrollar dichas competencias.

\section{Metodología}

Es una investigación cuantitativa no experimental transeccional, con alcance correlacional comparativo, porque tiene como finalidad determinar la relación entre dos o más variables (Hernández, Fernández y Baptista, 2014); en este caso, el uso de las TIC con algunos factores como el sexo de los participantes, el tener una computadora, o no, y acceso a internet.

\section{Participantes}

Los participantes fueron estudiantes de quinto y sexto de primaria de cinco escuelas públicas del municipio de Cajeme; utilizamos un muestreo no probabilístico por conveniencia y seleccionamos una institución por cada clasificación: dos escuelas federales, una estatal, una del Consejo Nacional de Fomento Educativo (Conafe) y una indígena. Dichas escuelas tienen una población

\section{Los participantes fueron}

\section{estudiantes de quinto y sexto}

\section{de primaria de cinco escuelas}

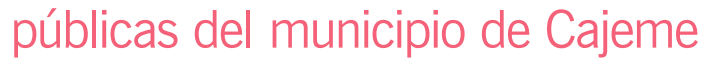

[...] dos escuelas federales, una estatal, una del Consejo Nacional de Fomento Educativo (Conafe) y total de 432 niños de entre once y doce años de edad; elegimos por escuela a un grupo de quinto y uno de sexto y la muestra quedó integrada por 201 participantes, de los cuales $64.8 \%$ estaban inscritos en las escuelas federales; 29\%, en la estatal; 2.6\%, en la del Conafe; y $3.6 \%$, en la indígena. Los alumnos de quinto representaron $51.6 \%$ y los de sexto, 48.4; en cuanto al sexo, 52.6 eran niños y $47 \cdot 4$, niñas.

\section{Instrumentos}

Diseñamos dos instrumentos y obtuvimos la sustentabilidad empírica de cada uno de ellos por medio de un análisis factorial confirmatorio. Estos instrumentos se derivaron de los elaborados por investigadores que participaron en el proyecto de investigación "Estudio comparativo del desarrollo de competencias digitales en el marco del programa Mi Compu.Mx", financiado por el Consejo Nacional de Ciencia y Tecnología (Conacyt), y fueron creados de acuerdo con el Programa de Habilidades Digitales para Todos (SEP, 2011), basado en los estándares propuestos por la ISTE (2007).

El primer instrumento es sobre el uso de las TIC en la vida diaria y consta de once ítems agrupados en una estructura unidimensional que miden el empleo de las TIC en general (ejemplo: puedo instalar y desinstalar un programa o software). Se contestó mediante una escala tipo Likert con cinco opciones de respuesta: 1 (nunca), 2 (raras veces), 3 (regularmente), 4 (casi siempre) y 5 (siempre). Los índices de ajustes obtenidos en el análisis factorial confirmatorio (X2 = 49.602, $\mathrm{p}=.052 ; \mathrm{CMIN}=1.41 ; \mathrm{CFI}=.97$; RMSEA $=.047$ ) demuestran la sustentabilidad empírica del modelo de medición antes descrito, es decir, hay congruencia entre el modelo teórico y el empírico (Cea, 2004). 
El otro instrumento se relaciona con el uso de las TIC en la escuela; consta de dos dimensiones: uso de las TIC para la comunicación en la escuela, conformado por tres ítems (ejemplo: uso el correo, el WhatsApp o el Facebook para comunicarme con mis compañeros de la escuela), y uso de las TIC en la escuela, con siete ítems (ejemplo: utilizo materiales digitales, como mapas, audiolibros y videos, para resolver tareas). Se contestó mediante una escala tipo Likert con cinco opciones de respuesta: 1 (nunca), 2 (raras veces), 3 (regularmente), 4 (casi siempre) y 5 (siempre). Los índices de ajustes obtenidos en el análisis factorial confirmatorio (X2 = $55.331, \mathrm{p}=.012 ; \mathrm{CMIN}=1.627$; $\mathrm{CI}=.97$; RMSEA $=.057)$ demuestran la sustentabilidad empírica del modelo de medición antes descrito.

\section{Procedimiento}

Con el propósito de recolectar información, solicitamos permiso a las autoridades académicas de cada institución educativa, quienes, además, brindaron orientación para la mejor forma de pedir a los profesores la participación de sus alumnos en el estudio; posteriormente, realizamos visitas a los diferentes planteles para recoger información.

A fin de obtener las propiedades psicométricas de los instrumentos, establecimos evidencias de validez de constructo mediante un análisis factorial confirmatorio. Respecto a los resultados, efectuamos análisis descriptivos por instrumento y pruebas t de Student para determinar si existen diferencias en las variables propuestas. Es importante aclarar que estos datos solo son válidos para nuestra muestra y no se pueden generalizar a otras poblaciones.

\section{... encontramos que los}

\section{estudiantes hacen un uso}

\section{moderado de las TIC en su vida}

\section{diaria, [...] [así como] para la comunicación en la escuela.}

\section{RESULTADOS}

\section{Uso de las TIC en la vida diaria}

En esta primera dimensión encontramos que los estudiantes hacen un uso moderado de las TIC en su vida diaria, con una media de 3.23 y una desviación estándar de 1.10. Con la intención de determinar si existen diferencias entre el sexo, realizamos una prueba $t$ de Student que arrojó que no existen diferencias entre niños y niñas. Lo anterior significa que las competencias digitales no varían según el sexo. Sin embargo, si hay diferencias en cuanto a contar con internet o no; aplicamos una prueba $t$ de Student, que reveló diferencias significativas (ver tabla 1). Para conocer si existen diferencias entre los estudiantes que cuentan con su computadora o tableta electrónica, o no, recurrimos a la misma prueba estadística anterior y el resultado indica que no existen diferencias significativas.

\section{Uso de las TIC en la escuela}

En cuanto a las dos dimensiones del uso de TIC en la escuela, también encontramos que los estudiantes hacen un empleo moderado de las TIC para la comunicación en la escuela al obtener una media de 3.20 y una desviación estándar de 1.44. En la segunda dimensión, de igual forma, hacen un uso 
moderado de las TIC en la escuela, con una media de 3.17y una desviación estándar de 1.09. Como en el instrumento anterior, llevamos a cabo una prueba t de Student para conocer si existen diferencias estadísticamente significativas entre las variables; el resultado fue que no hay diferencias significativas entre niños y niñas. Sin embargo, sí existen diferencias significativas entre alumnos que cuentan con acceso a internet, o no (ver tabla 1). En consecuencia, observamos que entre contar con acceso a internet, o no, las competencias en el uso de las TIC del alumno sí varían.

Asimismo, realizamos una prueba $t$ de Student para determinar si existen diferencias entre los estudiantes que cuentan con computadora o tableta electrónica, o no; solamente encontramos diferencias significativas en la dimensión sobre el uso de las TIC en la escuela (ver tabla 2).

A fin de determinar si existen diferencias entre los grupos de quinto y sexto grado, aplicamos una prueba t de Student mediante la cual obtuvimos que sí existen diferencias significativas, pero solo en la dimensión del uso de las TIC en la escuela (ver tabla 3). Lo anterior significa que los alumnos de más pequeños se perciben más competentes en el uso de las TIC en la escuela, es decir, utilizan más estas para actividades académicas que los alumnos de sexto. Esto puede deberse a que para los estudiantes de quinto grado es una novedad emplear las computadoras portátiles o tabletas electrónicas con propósitos académicos, y puedan acceder a los diferentes

Tabla 1. Diferencia entre estudiantes que cuentan con acceso a internet, o no, en su casa.

\begin{tabular}{|c|c|c|c|c|c|c|c|}
\hline & \multicolumn{2}{|c|}{$\begin{array}{l}\text { Sí CUENTO CON ACCESO A INTERNET } \\
\qquad N=118\end{array}$} & \multicolumn{2}{|c|}{$\begin{array}{l}\text { NO CUENTO CON ACCESO A INTERNET } \\
\qquad N=74\end{array}$} & \multirow[b]{2}{*}{ d } & \multirow[b]{2}{*}{$p$} & \multirow{2}{*}{$\begin{array}{c}\text { Cohen's } \\
\mathrm{d}\end{array}$} \\
\hline & $M$ & $\mathrm{DE}$ & $\mathrm{M}$ & $\mathrm{DE}$ & & & \\
\hline Uso de las TIC en la vida diaria & 3.40 & 1.07 & 2.96 & 1.09 & 2.76 & .00 & .40 \\
\hline $\begin{array}{l}\text { Uso de las TIC para la comunicación } \\
\text { en la escuela }\end{array}$ & 3.74 & 1.25 & 2.34 & 1.29 & 7.40 & .00 & 1.07 \\
\hline Uso de las TIC en la escuela & 3.30 & 1.06 & 2.98 & 1.11 & 1.97 & .05 & .28 \\
\hline
\end{tabular}

Tabla 2. Diferencias entre alumnos que cuentan con computadora o tableta electrónica, o no.

\begin{tabular}{|c|c|c|c|c|c|c|c|}
\hline & \multicolumn{2}{|c|}{$\begin{array}{c}\text { Sí CUENTO CON } \\
\text { COMPUTADORA } 0 \text { TABLETA } \\
\mathrm{N}=162\end{array}$} & \multicolumn{2}{|c|}{$\begin{array}{c}\text { No CUENTO CON COMPUTADORA } \\
\text { O TABLETA } \\
\mathrm{N}=30\end{array}$} & \multirow[b]{2}{*}{ d } & \multirow[b]{2}{*}{$\mathrm{p}$} & \multirow{2}{*}{$\begin{array}{c}\text { COHEN'S } \\
\mathrm{d}\end{array}$} \\
\hline & M & $\mathrm{DE}$ & M & $\mathrm{DE}$ & & & \\
\hline Uso de las TIC en la vida diaria & 3.28 & 1.09 & 2.96 & 1.10 & 1.45 & .14 & \\
\hline Uso de las TIC para la comunicación en la escuela & 3.21 & 1.42 & 3.12 & 1.52 & .32 & .74 & \\
\hline Uso de las TIC en la escuela & 3.24 & 1.08 & 2.82 & 1.10 & 1.94 & .05 & .28 \\
\hline
\end{tabular}

Tabla 3. Diferencias entre los alumnos de quinto y sexto grado.

\begin{tabular}{|l|c|c|c|c|c|c|c|}
\hline \multirow{2}{*}{} & \multicolumn{2}{|c|}{ QuinTo N=99 } & \multicolumn{2}{c|}{ SEXTO N= 93 } & & & COHEN'S \\
\cline { 2 - 10 } & $\mathrm{M}$ & $\mathrm{DE}$ & $\mathrm{M}$ & $\mathrm{DE}$ & $\mathrm{t}(190)$ & $\mathrm{p}$ & $\mathrm{d}$ \\
\hline Uso de las TIC en la vida diaria & 3.27 & 1.18 & 3.18 & 1.00 & .56 & .57 & \\
\hline Uso de las TIC para la comunicación en la escuela & 3.05 & 1.51 & 3.35 & 1.34 & -1.46 & .14 & \\
\hline Uso de las TIC en la escuela & 3.41 & 1.11 & 2.92 & 1.01 & 3.20 & .00 & .46 \\
\hline
\end{tabular}


materiales didácticos digitales; por lo tanto, están motivados y se sienten más competentes que los alumnos de sexto grado, para quienes el uso de las TIC en la escuela ya no es una novedad.

Además, en las pruebas de hipótesis, realizamos el análisis de lo que en estadística se conoce como tamaño del efecto, esto es, la probabilidad de rechazar una hipótesis nula que realmente es verdadera o de aceptar una hipótesis alternativa como verdadera cuando no lo es. El tamaño de las diferencias a través de la prueba de Cohen's son de un nivel que sobrepasa al pequeño y al grande, por lo cual cumplen con el criterio para afirmar que las diferencias entre las medias de los grupos no son falsas (Cárdenas y Arancibia, 2014).

\section{DISCUSIÓN}

En esta investigación indagamos la percepción sobre el uso de las TIC por parte de los estudiantes de quinto y sexto de primaria, quienes utilizan las computadoras y tabletas electrónicas en el aula; encontramos que estos hacen uso moderado de las TIC en general y para la comunicación en la escuela. Estos resultados no coinciden con los hallazgos obtenidos por Quintana, Cortada y Riera (2012), en el sentido de que los alumnos que tienen un mayor contacto con las TIC saben cómo usar la tecnología, muestran un mejor conocimiento y control de la computadora, así como mayores habilidades de alfabetización web, pero no para la búsqueda de información en internet.

Asimismo, los hallazgos de Chávez, Cantú y Rodríguez (2016) no coinciden con los de este estudio, ya que dichos autores encontraron que los alumnos reportan niveles en la media alta en sus competencias digitales y en el manejo o tratamiento de la información, y en nuestra investigación fue un nivel moderado. Por otra parte, los resultados de Fajardo, Villalta y Salmerón (2016) concuerdan con los obtenidos, ya que en ambos estudios los alumnos presentaron dificultades en numerosas habilidades digitales básicas. De la misma forma, las conclusiones de Mortis et al. (2014) son semejantes a las de esta investigación, ya que los alumnos perciben positivamente su conocimiento y uso de TIC para la búsqueda y el tratamiento de la información, así como en la comunicación social y el aprendizaje colaborativo.

Otro de los descubrimientos de este estudio es que no existen diferencias significativas entre los niños y las niñas en el uso de las TIC en general y en la escuela. Estos resultados coinciden con los de Volman et al. (2005), quienes investigaron la accesibilidad y el atractivo de los diferentes tipos de aplicaciones de las TIC en la educación para niñas y niños, y concluyeron que había una pequeña diferencia en cuanto a género, no significativa, sobre todo en estudiantes de educación primaria. En contraste, no coinciden con los resultados de Hatlevik (2012) y Aesaert y Van Braak (2015) con estudiantes de primaria, y de Mortis et al. (2014) con estudiantes de secundaria; en estos tres estudios se encontraron diferencias significativas entre niños y niñas respecto a sus competencias TIC; los niños se perciben menos competentes que las niñas. Lo anterior indica que las niñas están teniendo éxito en sus estudios mediante el uso de las computadoras en la escuela.

Respecto a otros resultados en este estudio, sí observamos diferencias significativas entre los estudiantes que cuentan con internet en su casa, o no, pero ninguna significativa entre los que tienen una computadora o tableta electrónica, o no. Esto coincide con las conclusiones de la investigación de Villegas, Mortis y Del Hierro en 2015, cuando encontraron que las competencias digitales de los alumnos de quinto y sexto de primaria (que cuentan con una tableta electrónica o una computadora portátil como beneficiarios del proyecto Mi Compu.Mx) sí variaban dependiendo de su acceso a internet desde su casa.

Por otra parte, estos resultados no coinciden con los de Mortis et al. (2014), quienes advirtieron que el poseer una computadora es un factor determinante respecto al nivel de las competencias tecnológicas de los alumnos de educación básica, 
... los alumnos de quinto

\section{y sexto grado se perciben}

\section{igual de competentes en las}

dos primeras dimensiones:

uso de las TIC para la vida diaria y uso de las TIC para la comunicación en la escuela.

específicamente de secundaria. Sin embargo, sí concuerdan con las conclusiones de Villegas, Mortis y Del Hierro (2015), que explican que no hallaron diferencias significativas en las competencias digitales de los estudiantes que cuentan con computadora portátil $\mathrm{mx}$ o tableta, o no; quizás esto se deba a que los niños en su casa manejan computadora de escritorio, portátil o una tableta electrónica de algún familiar.

En referencia a esta conclusión, propuesta por Villegas, Mortis y Del Hierro (2015), puede aplicarse a la situación de los estudiantes que participaron en este estudio, porque la mayoría de los niños tienen acceso a alguna computadora o tableta en sus casas o las de sus amigos, ya que por lo general las emplean para actividades lúdicas. Ortiz, Peñaherrera y Ortega (2012), quienes efectuaron un estudio de caso para describir y analizar en profundidad el uso de las TIC de los estudiantes de primaria, encontraron que estos utilizan la computadora principalmente para el juego, después para trabajos en clase $\mathrm{y}$, por último, para navegar en internet.

Los alumnos de quinto y sexto grado se perciben igual de competentes en las dos primeras dimensiones: uso de las TIC para la vida diaria y uso de las TIC para la comunicación en la escuela. Esto no coincide con los hallazgos de Fajardo, Villalta y Salmerón (2016), quienes señalan que los estudiantes de tercero de secundaria obtuvieron mayores puntuaciones que los de quinto de primaria en las habilidades digitales. Sin embargo, en la dimensión sobre el uso de las TIC en la escuela, los alumnos de quinto grado se perciben más competentes que los de sexto; es decir, los alumnos más pequeños utilizan en mayor medida las TIC para actividades académicas y, por lo tanto, se perciben más competentes que los alumnos más grandes.

Estos resultados podrían equipararse con los alcanzados por Fajardo, Villalta y Salmerón (2016) en cuanto a los usos de internet, que revelan que los estudiantes de primaria lo utilizan mayormente para actividades académicas: buscar información (80\%), seguido de actividades lúdicas: jugar en línea (60\%), descargar música (40\%) y ver videos (40\%); en cambio, los de secundaria recurren a internet más para socializar y actividades lúdicas: redes sociales (90\%), descarga de música (90\%), ver videos (90\%) y juegos en línea (80\%) que para labores académicas: búsqueda de información (80\%) y lectura del correo electrónico (70\%).

\section{CONCLUSIONES}

Podemos afirmar que la integración de las TIC a las aulas es muy importante, ya que implican un cambio en el modelo educativo, que significa pasar de uno centrado en la enseñanza a otro que gire en torno al aprendizaje. La incorporación de las TIC en las aulas de educación primaria demanda no solo incluir mejores recursos educativos, más atractivos y motivantes, sino propiciar la adquisición de algunas 
competencias digitales y la habilidad de procesamiento de información, y sobre todo un mejor desarrollo cognitivo gracias a la función informativa, transmisora e interactiva de los recursos TIC (Area, 2009; Gómez y Macedo, 2010; Castañeda, Carrillo y Quintero, 2013).

De esta manera, podemos reflexionar acerca de la necesidad de profundizar en algunas líneas de investigación sobre la forma de orientarse con recursos didácticos en internet para favorecer la promoción de las competencias en el uso de las TIC, ya que la expectativa de la incorporación de estas en la educación básica es que propicien una mejora en la comunicación, construcción del conocimiento, procesamiento de información y desarrollo cognitivo. En la medida que nos acerquemos a la realidad de nuestros estudiantes, los procesos educativos con uso de las TIC permitirán logros significativos en sus aprendizajes que, a su vez, les ayudan a hacer frente a los retos actuales y futuros de la sociedad de la información y el conocimiento.

Recomendamos que en futuros programas para fomentar una cultura de ciudadanía digital en los diferentes estados de la república mexicana, además de equipar a las escuelas de educación básica y proporcionar computadoras a estudiantes de primaria, se realicen diagnósticos previos para medir los conocimientos y las habilidades en el uso de las TIC por parte de docentes y estudiantes. Además, proponemos que se impartan cursos de capacitación a los profesores para lograr un mejor uso pedagógico de ellas en cuanto a herramienta educativa y, de igual modo, a los padres de familia para lograr el empleo adecuado en sus hogares.

Asimismo, es conveniente que en futuros estudios se mida el desarrollo de la competencia digital en los profesores, ya que es una figura fundamental para el aprendizaje

\section{En la medida que nos}

\section{acerquemos a la realidad}

\section{de nuestros estudiantes, los}

\section{procesos educativos con uso}

de las TIC permitirán logros

significativos en sus aprendizajes

que, a su vez, les ayudan a

hacer frente a los retos actuales

y futuros de la sociedad de la

información y el conocimiento.

del estudiante, y que se realicen investigaciones que abarquen todas las competencias digitales en los estudiantes, que pueden ser las propuestas por el ISTE, de preferencia las más actualizadas, debido a que los resultados de nuestro estudio fueron obtenidos mediante instrumentos de autorreporte, que miden solo la opinión de los estudiantes sobre su competencia en el uso de las TIC.

\section{Agradecimientos}

Reconocemos el apoyo recibido para el proyecto de investigación "Estudio comparativo del desarrollo de competencias digitales en el marco del programa Mi Compu.Mx", financiado por el Conacyt, y para el proyecto "Competencias digitales en alumnos de primaria”, financiado por el PROFAPI 2016-0018 del Instituto Tecnológico de Sonora. Los resultados de nuestro estudio fueron obtenidos gracias al financiamiento de ambas instituciones. a 


\section{REFERENCIAS BIBLIOGRÁFICAS}

Aesaert, Koen \& Van Braak, Johan. (2015). Gender and socioeconomic related differences in performance based ICT competences. Computers \& Education, vol. 84, pp. 8-25. http:// dx.doi.org/10.1016/j.compedu.2014.12.017

Area, Manuel. (2009). Introducción a la tecnología educativa. Islas Canarias, España: Universidad de La Laguna. Recuperado de https://campusvirtual.ull.es/ocw/file.php/4/ebookte.pdf

Cárdenas, Manuel y Arancibia, Héctor. (2014). Potencia estadística y cálculo del tamaño del efecto en $\mathrm{G}^{*}$ power: complementos a las pruebas de significación estadística y su aplicación en psicología. Salud y Sociedad, vol. 5, núm. 2, pp. 210224. Recuperado de https://dialnet.unirioja.es/servlet/ articulo? codigo $=4945415$

Castañeda, Arturo; Carrillo, Jesús; Quintero, Zaira. (2013). El uso de las TIC en la educación primaria: la experiencia Enciclomedia. México: Redie.

Cea, María. (2004). Análisis multivariable. Teoría y práctica en la investigación social. Madrid: Síntesis.

Chávez, Flor; Cantú, Maricarmen; Rodríguez, Catalina. (2016). Competencias digitales y tratamiento de información desde la mirada infantil. Redie, vol. 18, núm. 1, pp. 209-220. Recuperado de http://redie.uabc.mx/redie/article/view/631

Díaz, Diana; Rodríguez, Monserrat; Sánchez, Wendy; Rivera, Nohemi; Ramírez, María. (2015). Competencias digitales en el marco del programa Mi Compu.Mx: estudio piloto en Colima, Sonora y Tabasco. Memorias del Segundo Congreso Internacional de Innovación Educativa, México, DF. Recuperado de http:// repositorio.itesm.mx/ortec/handle/11285/575398

García, Ramona; Angulo, Joel; Cuevas, Omar. (2015). Mi Compu.Mx: opinión de padres de familia, docentes y directivos sobre su aplicación y desarrollo. Memorias del XIII Congreso Nacional de Investigación Educativa, Chihuahua, México. Recuperado de http://hdl.handle.net/11285/575957

Gómez, Luz y Macedo, Julio. (2010). Importancia de las TIC en la educación básica regular. Investigación Educativa, vol. 14, núm. 25, pp. 209-226. Recuperado de http://sisbib.unmsm. edu.pe/bibvirtual/publicaciones/inv_educativa/2010_n25/pdf/ a12v14n25.pdf

European Commission. (2007). Key competences for lifelong learning. European reference framework. Luxembourg: Office for Official Publications of the European Communities. Recuperado de https://www.erasmusplus.org.uk/file/272/download
Fajardo, Inmaculada; Villalta, Ester; Salmerón, Ladislao. (2016). ¿Son realmente tan buenos los nativos digitales? Relación entre habilidades digitales y la lectura digital. Anales de Psicologías, vol. 32, núm. 1, pp. 89-97. Recuperado de https://dialnet.unirioja.es/servlet/articulo?codigo $=5292592$

Hatlevik, Ove. (2012). Analyzing factors influencing students productive use of computers: A structural equation model. The International Journal of Technology, Knowledge and Society, vol. 7, núm. 4, pp. 11-27. Recuperado de http://eds.b.ebscohost. com/eds/pdfviewer/pdfviewer?sid =81c39e63-0eea-4ff495d1-773c8c7a7ced\%40sessionmgr103\&vid = 0\&hid $=120$

Hernández, Roberto; Fernández, Carlos; Baptista, Pilar. (2014). Metodología de la investigación educativa. México: McGraw-Hill.

International Society for Technology in Education (ISTE). (2007). ISTE Standards students. Recuperado de http://www.iste.org/standards/iste-standards/standards-for-students

McKenney, Susan \& Voogt, Joke. (2010). Technology and young children: How 4-7 year olds perceive their own use of computers. Computer in Human Behavior, vol. 26, núm. 4, pp. 656-664. http://dx.doi.org/10.1016/j.chb.2010.01.002

Mortis, Sonia; Cuevas, Omar; García, Imelda; Cabero, Julio. (2014). Competencias tecnológicas en alumnos de secundaria. En Sonia Echeverría, María Fernández, Eneida Ochoa y Dora Ramos (comps.). Ambientes de aprendizaje y contexto de desarrollo social (pp. 117-119). México: Pearson.

National Educational Technology Standards for Students (NETS-S). (2007). Estándares nacionales (EEUU) de tecnologías de información y comunicación (TIC) para estudiantes: la próxima generación. Recuperado de https://www.iste.org/docs/pdfs/ nets 2007 spanish.pdf?sfvrsn $=2$

Ortiz, Ana; Peñaherrera, Mónica; Ortega, Juana. (2012). Percepciones de profesores y estudiantes sobre las TIC. Un estudio de caso. Edutec-e, núm. 41, pp. 1-15. http://dx.doi.org/10.21556/ edutec.2012.41.352

Parlamento Europeo. (2009). Competencias clave para el aprendizaje permanente. Recuperado de http://www. europarl.europa.eu/meetdocs/2004_2009/documents/ am/609/609485/609485es.pdf

Quintana, María; Cortada, Meritxell; Riera, Jordi. (2012). Internet navigation and information search strategies: How do children are influenced by their participation in an intensive ICT project. International Journal of Technology \& Design Education, vol. 22, núm. 4, pp. 513-529. http://dx.doi.org/10.1007/s10798011-9158-4 
Revuelta, Francisco. (2011). Competencia digital: desarrollo de aprendizajes en mundos vitales en la escuela 2.0. Revista Electrónica de Tecnología Educativa, núm. 37, pp. 1-14. http:// dx.doi.org/10.21556/edutec.2011.37.397

Rocha, María y Ramírez, María. (2015). Los sujetos y objetos que inciden en el desarrollo de competencias digitales en el marco del programa Mi Compu.Mx: caso escuela rural de Colima. Memorias del XIII Congreso Nacional de Investigación Educativa, Chihuahua, México. Recuperado de http://hdl.handle. net/11285/575878

Secretaría de Educación Pública (SEP). (2011). Acuerdo número 592 por el que se establece la articulación de la educación básica. Recuperado de https://www.sep.gob.mx/work/models/ sep1/Resource/9721849d-666e-48b7-8433-0eec1247f1ab/ a592.pdf

Secretaría de Educación Pública (SEP). (2013). Dotación de equipos de cómputo portátiles para niños de quinto y sexto grados de escuelas primarias públicas. Recuperado de http://www.basica. primariatic.sep.gob.mx/descargas/IIC_DOTACION_BAIA.pdf

Trigueros, Francisco; Sánchez, Raquel; Vera, María. (2012). El profesorado de educación primaria ante las TIC: realidad y retos. REIFOP, vol. 15, núm. 1, pp. 101-112. Recuperado de http://www.aufop. com/aufop/uploaded files/articulos/1335399123.pdf

Villegas, Marisol; Mortis, Sonia; Del Hierro, Elizabeth. (2015). Competencias digitales de alumnos de educación primaria participantes en el proyecto Mi Compu.Mx. En Raquel García, Sonia Mortis, Jesús Tánori y Teresa Sotelo (comps.). Educación y salud: evidencias y propuestas de investigación en Sonora (pp. 152-165). México: Fontamara.

Volman, Monique; Van Eck, Edith; Heemskerk, Irma; Kuiper, Els. (2005). New technologies, new differences. Gender and ethnic differences in pupils' use of ICT in primary and secondary education. Computers \& Education, vol. 45, núm. 1, pp. 35-55. http://dx.doi.org/10.1016/j.compedu.2004.03.001

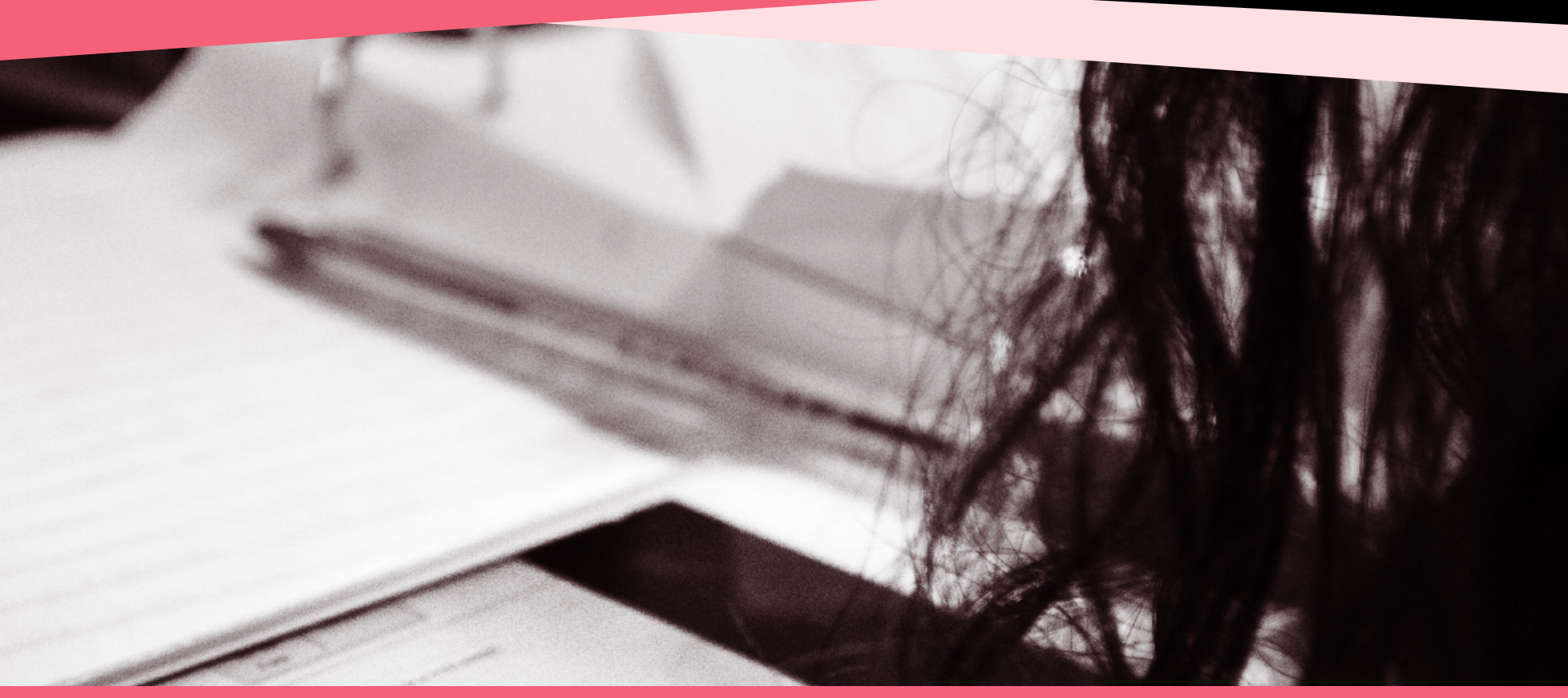

"Este artículo es de acceso abierto. Los usuarios pueden leer, descargar, distribuir, imprimir y enlazar al texto completo, siempre y cuando sea sin fines de lucro y se cite la fuente".

\section{CÓMO CITAR ESTE ARTÍCULO:}

Villegas Pérez, Marisol; Mortis Lozoya, Sonia Verónica; García López, Ramona Imelda y Del Hierro Parra, Elizabeth. (2016). Uso de las TIC en estudiantes de quinto y sexto grado de educación primaria. Apertura, 9 (1), pp. 50-63. http://dx.doi.org/10.32870/Ap.v9n1.913 